\title{
Inadequate HIV care after incarceration: case closed
}

Published Online November 27, 2017 http://dx.doi.org/10.1016/ S2352-3018(17)30210-2
At any given moment, one in 99 adults in the USA are behind bars in prisons and jails, often for offenses related to the use or sale of illicit substances. ${ }^{1}$ This mass incarceration overwhelmingly affects racial and ethnic minority men; the lifetime risk of incarceration is more than 30\% for African-American men, compared with $17 \%$ for white men. ${ }^{2}$ Drug laws that strictly penalise the possession, use, and sale of illicit substances, and the less overt targeting of minorities for search and arrest, have led to people with HIV infection also being disproportionately incarcerated.

Most metrics show that HIV care in US prisons is adequate; in jails, where lengths of stay are typically short and local budgets are typically meager, the quality of HIV care is more variable. However, after release, former inmates often have loss of viral suppression or loss of medical care. ${ }^{3-5}$ In their Article in The Lancet HIV ${ }^{6}$ Kelsey B Loeliger and colleagues add a weighty analysis to the mounting evidence on the perils of release from incarceration for people with HIV. By linking administrative and medical databases maintained by the Connecticut state correctional system with state public health authority data on community-based testing for HIV, Loeliger and colleagues assessed linkage to care after release from prisons and jails between 2007 and 2014, using viral load measurements as a proxy for engagement in HIV care.

The authors found low rates of linkage to care in the weeks just after release, when the antiretroviral supply provided by correctional facilities would run out, and viral load tests were done within 30 days of release in only a third of post-release periods. Encouragingly, evidence of engagement in HIV care increased over time; $76 \%$ of releases had a viral load drawn within 6 months after release. However, a substantial proportion had detectable viral loads of HIV. Factors independently associated with linkage to care within
30 days of release included intermediate incarceration duration (31-364 days), receipt of transitional case management, diagnosis with two or more comorbidities, and treatment with antiretroviral therapy during incarceration.

This report has several important limitations, including those that are inherent to the reliance on data sources that might be incomplete and the use of an imperfect surrogate for actual visitation to a HIV care provider. However, the findings are similar to that of previous reports and highlight that the HIV/AIDS strategy in the USA is negligent and inadequate, given the consequences that a fumbled transition of HIV care after release from prison and jail could have for individual and public health.

In addition to the risk of CD4 cell depletion and immunosuppression, breaks in HIV therapy lead to a rebound in viraemia, and deleterious immune activation and inflammatory responses. The discontinuation of antiretrovirals in the SMART trial of treatment interruption guided by $C D 4$ cell count was associated with allcause mortality and opportunistic conditions. ${ }^{8}$ Because of the risks associated with viral rebound, studies involving analytic treatment interruption now have safety measures to limit the magnitude and duration of viraemia.

Equally concerning is the potential loss of viral suppression after release, which could lead to an increased risk of transmission of HIV to other people. ${ }^{9}$ A return to sexual and injection behaviours that risk viral transmission, coupled with loss of viral suppression will contribute to the spread of HIV in communities that are affected disproportionately by sexually transmitted infections, substance use, and incarceration.

The authors also found, but do not stress, that even during the relatively controlled setting of incarceration, the proportion of incarceration periods that involved 
treatment with antiretrovirals was low, and the proportion that achieved viral suppression was even lower. Although rates of both of these factors improved over time, only $56 \cdot 3 \%$ of the releases during 2013-14 had an undetectable viral load before release. Notably, this finding suggests access and utilisation of HIV therapy in correctional settings remains suboptimal, particularly because of the reported association between subsequent linkage to care and the receipt of HIV therapy during incarceration.

The authors, like others, suggest ways to solve the inadequacy of post-release care, including real-time use of public health surveillance data to identify and intervene with those out of care (ie, Data to (are) ${ }^{10}$ and broader use of case management type services. Data to Care is an important public health tool; however, it primarily acts as a safety net designed to catch those who fall out of care. Case management is a pragmatic and attractive upstream intervention but, its effectiveness in linking people with HIV to care and promoting viral suppression is unclear, and even in studies with positive findings, effects have been modest. ${ }^{11,12}$

The intransigence of the break in the continuum of HIV care when inmates transition from incarceration to the community-despite approaches such as case management and motivational interventions-shows that structural barriers hinder successful community reintegration after release from prison or jail. Poverty and food insecurity, untreated substance use and mental health disorders, racial and ethnic discrimination, and other prevalent social factors, are inadequately addressed by even the most thoughtfully designed individual-level interventions. Recognition of these social factors, including mass incarceration itself, as being toxic and unacceptable will spur on the policy changes that are needed to bridge this tragic gap in HIV care.

\section{${ }^{*}$ David Alain Wohl, David Loren Rosen}

Institute of Global Health and Infectious Diseases, The University of North Carolina at Chapel Hill, Chapel Hill, NC 27514, USA wohl@med.unc.edu

1 Wagner P and Rabuy B. Mass incarceration: the whole pie 2017. Prison policy initiative. March 14, 2017. https://www.prisonpolicy.org/ reports/pie2017.html (accessed Sept 29, 2017)

2 Mauer M. Addressing racial disparities in incarceration. Prison J 2011 91 (suppl: 3) 87S-101S

3 Baillargeon J, Giordano TP, Rich JD, et al. Accessing antiretroviral therapy following release from prison. JAMA 2009; 301: 848-57.

4 Springer SA, Pesanti E, Hodges J, Macura T, Doros G, Altice FL. Effectiveness of antiretroviral therapy among HIV-infected prisoners: reincarceration and the lack of sustained benefit after release to the community. Clin Infect Dis 2004; 38: 1754-60.

5 Stephenson BL, Wohl DA, Golin CE, Tien HC, Stewart P, Kaplan AH. Effect of release from prison and re-incarceration on the viral loads of HIV-infected individuals. Public Health Rep 2005; 120: 84-88.

6 Loeliger KB, Altice FL, Desai MM, Ciarleglio MM, Gallagher C, Meyer JP. Predictors of linkage to HIV care and viral suppression levels following release from jails and prisons: a retrospective cohort study. Lancet HIV 2017; published online Nov 27. http://dx.doi.org/10.1016/S23523018(17)30209-6

7 Cozzi-Lepri A, French MA, Baxter J, et al. Resumption of HIV replication is associated with monocyte/macrophage derived cytokine and chemokine changes: results from a large international clinical trial. AIDS 2011; 25: 1207-17.

8 El-Sadr WM, Lundgren JD, Neaton JD, et al. CD4 + count-guided interruption of antiretroviral treatment. N EnglJ Med 2006; 355: 2283-96.

9 Hamlyn E, Ewings FM, Porter K, et al. Plasma HIV viral rebound following protocol-indicated cessation of ART commenced in primary and chronic HIV infection. PLoS One 2012; 7: e43754.

10 Centers for Diseases Control and Prevention. Using HIV surveillance data to support the HIV care continuum. Effective interventions: HIV prevention that works. Data to care. 2017. https://effectiveinterventions.cdc.gov/en/ HighlmpactPrevention/PublicHealthStrategies/DatatoCare.aspx (accessed Sept 29, 2017)

11 Zaller ND, Holmes L, Dyl AC, et al. Linkage to treatment and supportive services among HIV-positive ex-offenders in Project Bridge. J Health Care Poor Underserved 2008; 19: 522-31.

12 Wohl DA, Golin CE, Knight K, et al. Randomized controlled trial of an intervention to maintain suppression of HIV viremia after prison release: the imPACT trial. J Acquir Immune Defic Syndr 2017; 75: 81-90. 